\title{
Index auteurs
}

\section{Author's index}

Vol. 29 - 2013

C) Springer-Verlag France 2014

Afifi M.A., 19

Almoubaker S., 77

Aloulou I., 60

Ammoumri O., 49

Arroud M., 19

Atarraf K., 19

Ayadi K., 35

Azagui Y., 90

Belkhou A., 73

Bellan D., 68

Ben Kridis W., 45

Ben Salah F.Z., 60

Benabid M., 49

Benamou P.-H., 2, 5, 33

Benchebba D., 23

Benhaddou H., 85

Benhima M.A., 73

Bouabid S., 23

Boudard, G., 68

Boudawara T., 45

Bouhlal Y., 73

Bousselmame N., 23

Boutayeb F., 49, 77

Bouysset M., 1, 59

Chaffry B., 23

Chagar B., 23

Chakoura M., 14

Chater L., 19

Chaumont P.-L., 68

Cherrad T., 14

Coudane H., 68

Coughlin M.J., 7
Da S.C., 94

Dakouré P.W.H., 94

Daoud J., 45

Daoudi A., 98

Dedome D., 40

Delagoutte J.-P., 68

Diallo M., 94

Doty J.F., 7

Dziri C., 60

El Andaloussi Y., 90

Elleuch M., 60

Ellouz Z., 45

Feki J., 45

Fikry T., 73

Frikha M., 45

Galois L., 40

Gandéma S., 94

Goldzak M., 100

Haddoune A.R., 90

Ibn El Kadi K., 49

Kasmaoui H., 14

Keskes H., 35

Khanfir A., 45

Kharrat O., 60

Lahdidi S., 90

Lahrach K., 49, 77

Lebib S., 60

Louaste J., 14
Mainard D., 40

Marzouki A., 49, 77

Mezzani A., 49, 77

Miri I., 60

Mittlmeier T., 100

Naceur A., 35

Najeb Y., 73

Najib A., 98

Nespola A., 40

Ouarab M., 90

Ouertateni M., 53

Poircuitte J.M., 40

Pomares G., 68

Rachid K., 14

Saliou S., 49

Sellami J., 35

Simon P., 100

Soulama M., 94

Strugarek C., 40

Tlili N., 53

Toumi N., 45

Trigui M., 35

Yacoubi H., 98

Yahia A., 35

Zaddoug O., 23

Zejjari H., 14

Zribi M., 35

Zribi W., 35 